\title{
44. ASTRONOMICAL OBSERVATIONS FROM OUTSIDE THE TERRESTRIAL ATMOSPHERE
}

\author{
(OBSERVATIONS ASTRONOMIQUES AU-DEHORS \\ DE L'ATMOSPHËRE TERRESTRE)
}

PRESIDENT: A. D. Code

VICE-PRESIDENT: R. M. Bonnet

ORGANIZING COMmITTEE: J. E. Blamont, C. de Jager, A. H. Gabriel, R. Giacconi, P. Léna, K. A. Pounds, V. K. Prokof'ev, R. Tousey, R. Wilson.

Commission 44 is by title technique oriented. The following statement of the role of the commission was prepared for use by the General Secretary in responding to requests by non-astronomers with respect to the activities of IAU commissions.

'The Earth's atmosphere is opaque to radiation throughout most of the electromagnetic spectrum. The light reaching the surface of the Earth from celestial objects is confined primarily to the visual region of the spectrum and a larger window in the radio region. Much of the infrared and all the far ultraviolet, $\mathrm{X}$-ray and $\gamma$-ray radiation are absorbed in the upper atmosphere. Thus astronomers must carry their telescopes and auxiliary instrumentation above the Earth's atmosphere to unlock the clues on the structure of the universe revealed by the light at these wavelengths. Furthermore the light in the accessible region of the spectrum that does reach the surface of the Earth is distorted and scattered in such a manner that the spatial resolution and details of the radiating source is seriously degraded.

Commission 44 was initially organized to coordinate those efforts in astronomical investigations that utilize space crafts, sounding rockets, balloons and high altitude aircraft to carry out studies above the earth's atmosphere. While the scientific investigations are closely interlocked with disciplines covered by other IAU Commissions the techniques introduce unique problems of international cooperation justifying a commission whose responsibility is directed towards extraterrestrial observations.'

As space astronomy has matured it has lost its identity and simply taken its place among the variety of observational techniques employed by astronomers to advance our understanding of the physical universe. The scientific results of space astronomy span most of the areas of current astrophysical research. As such many of the scientific results obtained from rockets, satellites and space probes are to be found in the reports of other IAU Commissions.

In the commission report covering the period 1967-69 it was emphasized that the report 'could only be a selection of the most important advances during the past three years and not a complete review of the field'. This is, of course, even more true today. The report on the 1970-72 period took as its guidelines 'not to consider the scientific results, these being a matter for other IAU Commissions, but to mainly pay attention to the technical achievements and new possibilities of development'. In this report we shall adopt this general approach; however, even the technical aspects have become rather common and well known and we shall try to emphasize the operational aspects.

During the last three years there has been a much greater involvement of astronomers from all areas in the observational and theoretical investigations resulting from space astronomy. It is no longer true that space observations are carried out by a principle investigator flying his experiment. It is common to speak of space observatories and they have been characterized by significant international cooperation and the utilization of the facilities by mainly 'guest investigators'. In an attempt to reflect this wider participation several observatory reports will be presented here. These reports, however, represent, only a small sample of the current, on-going research in space astronomy. Many satellites have made important contributions to astronomy during the past three years. The report period began with the conclusion of operations of the 
first major stellar observatory in space, OAO-2, which after 50 months of operation failed and was turned off on 1973, February 13. To date over 150 scientific papers have been presented in ultraviolet spectrophotometric data obtained by the two experimental pack ages on OAO-2, and a large body of high quality data still remain to be discussed. The impact of the new discoveries provided by the X-ray satellite Uhuru and the high dispersion ultraviolet satellite Copernicus (OAO-3) were just making themselves felt three years ago. Today the results from these successful missions are numbered among the scientific highlights of the period. A large body of scientific data of quality comparable to traditional ground-based observations has been obtained by a variety of space craft. The ESRO astronomical satellite TD-1 A obtained extensive measurements in the ultraviolet. The OSO satellites continued to provide a means of investigating the complex structure of the Sun as well as providing a vehicle for other astronomical investigations. The results from the most recent solar observatory, OSO-8, and the ATM measurements carried out on Skylab are the topic of a joint commission meeting, at the General Assembly. Ultraviolet measurements have been obtained with the Orion-2 experiment aboard Soyuz-13, by the Netherlands Astronomical satellite, ANS, the French satellite Aura, and by measurements from Mariner 9. X-ray observations have been carried out with Uhuru, Copernicus, OSO-7, ANS, Ariel 5, SAS-3, and OSO-8, among others. To date investigations in the infrared have utilized rockets, balloons and high altitude aircraft to great profit and many other investigations have been conducted with these techniques. Accounts of the scientific results of some of these missions are presented in the reports of several other commissions in this volume. The discussion of ultraviolet interstellar lines observed by TD-1A and Copernicus has been prepared by D. C. Morton and presented in the report for IAU Commission 34 on Interstellar Matter and Planetary Nebulae. A report on ultraviolet stellar observations by L. Houziaux is to be found in the report of Commission 29 on Stellar Spectra, while Commissions 10 and 12 on Radiation and the Solar Atmosphere and on Solar Activity review the UV, EUV and X-ray results of the various solar satellite missions. A Joint Discussion on 'X-ray Binaries and Compact Objects' has been planned by Commissions 42, 44, and 48 for the General Assembly and some of the observational material is included in the reports of these Commissions.

Ultraviolet astronomy prior to this report period was reviewed by Bless and Code (1972); an account of some of the more recent advances is given in 'Reports of New Ultraviolet Observations' presented at the COSPAR 16th Plenary Meeting, 23 May-5 June 1973 and prepared by van der Hucht and Lamers and published in 'The Astronomical Institute at Utrecht Internal Report ROF72'.

Survey articles on some of the scientific and technical advances accruing from space astronomy may be found in the following references:

Culhane and Acton, Ann. Rev. Astron. Astrophys. 12, 359, 1974.

Spitzer and Jenkins, Ann. Rev. Astron. Astrophys. 13, 133, 1975.

Blumenthal and Tucker, Ann. Rev. Astron. Astrophys. 12, 23, 1974.

Silk, Ann. Rev. Astron. Astrophys. 11, 1269, 1973.

Peterson, Ann. Rev. Astron. Astrophys. 13, 423, 1975.

Phil. Trans. Roy. Soc. London A 279, 297, 1975.

In the X-ray region some 200 sources have been discovered in the 2 to $10 \mathrm{keV}$ range and positional accuracy has continued to improve so that many of the sources have optical identifications. The recognition of a class of binary X-ray sources has shed light on the emission mechanism for these objects, one component of which is most likely a collapsed object such as a neutron star, or in the case of Cygnus X-1, possibly a black hole. The discovery of X-ray sources in globular clusters with a higher frequency than among stars in the galactic plane has presented a fascinating problem. The suggestion has been put forth that the higher density in the globular cluster has led to an active event in the nucleus perhaps involving collapsed objects. X-rays from quasars and Seyferts are undoubtedly related to the energetic processes in the nuclei of such systems, while the X-ray emission in the direction of some clusters of galaxies may relate to the intergalactic medium. The diffuse X-ray background in the 2 to $10 \mathrm{keV}$ range is approximately isotropic, hence likely to be extragalactic, while the soft 
$\mathrm{X}$-ray background apparently is primarily galactic and suggests a hot $\left(\sim 10^{6} \mathrm{~K}\right)$, low density component in the interstellar medium. The observations of $\gamma$-ray bursts have presented entirely new phenomena that promise to be one of the exciting new areas of research in high energy astrophysics.

Important advances in research on the interstellar medium have been achieved by high resolution spectrophotometry of the ultraviolet interstellar absorption lines. The observation of relatively wide $O$ VI lines suggest a gas component with a temperature of the order of $2 \times 10^{5} \mathrm{~K}$ to $2 \times 10^{\circ} \mathrm{K}$ which may be consistent with the source of the soft X-ray background mentioned above. Determinations of the column densities of neutral hydrogen from measurements of interstellar $L y-\alpha$ yield number densities between $0.01 \mathrm{H} \mathrm{cm}^{-3}$ to $2.5 \mathrm{H} \mathrm{cm}^{-3}$. The $\mathrm{H} \mathrm{I}$ density is particularly low near the Sun and remains low for the stars studied in Orion, Canis Major and Puppis. These associations are characterized by little reddening and considerable H II. The bulk of the neutral hydrogen observed in $21-\mathrm{cm}$ is apparently beyond the Orion complex. The highest $\mathrm{H} \mathrm{I}$ densities are found from stars in Perseus, Ophiuchus and Scorpius. Models attempting to interpret the observed strengths of $\mathrm{HI}, \mathrm{H}_{2}, \mathrm{HD}$ and other interstellar lines indicate that the interstellar medium is characterized by sheets or small clumps of $\mathrm{HI}$ and involves shocks or tunnels resulting from supernovae or stellar winds. This picture is consistent with the large scale distribution of $21 \mathrm{~cm}$ radiation. Measurements of the $\mathrm{D} / \mathrm{H}$ ratio of the order of $2 \times 10^{-5}$ and upper limits on the B/D ratio suggest that deuterium was formed in the initial Big Bang and that the corresponding density required to produce the observed $\mathrm{D} / \mathrm{H}$ ratio is therefore too low to close the universe. The interstellar abundances of most of the elements studied relative to hydrogen, are significantly below solar abundance. The abundances range from nearly solar for $\mathrm{S}$ and $\mathrm{Zn}$ to about $2 \times 10^{-4}$ the solar abundance for $\mathrm{Ca}$. These depletion patterns are presumably due to the formation of grains although other processes must be operative as well. Interstellar extinction data provide one of the important constraints on the composition of the interstellar grains. To date ultraviolet interstellar extinction curves have been determined for several hundred stars using data from OAO-2, TD1-A, and ANS. Although the extinction shortward of $2000 \AA$ shows considerable variation, the $2200 \AA$ extinction bump is remarkably similar from star to star. Willis and Wilson have found an enhanced $2200 \AA$ absorption band in the expanding nebulosity NGC 6888. Borgman, Van Duinen and Koorneff have found no evidence for the $2200 \AA$ extinction feature in several regions close to 30 Doradus in the Large Magellanic cloud. These appear to be the most outstanding exceptions to the uniformity of this interstellar feature.

In solar astronomy large improvements in our understanding of the physical processes in active regions and flares have been made possible by a variety of measurements from space crafts. Measurements of energetic electrons, protons and heavier nuclei and their relationship with various electromagnetic emissions giving a more complete picture of solar phenomena have also characterized advances in solar physics. The discovery of coronal holes and bright points in far UV images have importance with respect to the general solar magnetic field and solar wind. One model for a coronal hole is that it may be a region where the solar magnetic field is open to the interplanetary medium. Conduction outward along the field lines causes a depletion of the corona in these regions. Solar astronomy can not help but benefit too from the accumulating data on the chromospheres and coronae of other stars. From analysis of Copernicus data, evidence for the presence of chromospheres has been found for stars as early as spectral type B as well as for later type, and atmospheric structural models have been constructed for a number of stars.

Stellar observations in the ultraviolet have provided fundamental data on stellar structure. In particular it has become possible to determine expirical bolometric corrections and effective temperatures for early-ty pe main sequence stars. The absolute calibration of stellar fluxes in the ultraviolet is now sufficiently reliable that it is possible to determine the energy radiated over most of the relevant spectral range to yield empirical bolometric corrections. Combining these data with measured angular diameters provides observationally determined effective temperatures. Ultraviolet measurements have also indicated that the number of hot subluminous stars in the general field and in globular clusters is greater than present luminosity functions would suggest. Recently observations in the EUV $(\sim 100 \AA)$ have been obtained of several stars 
indicating both the high temperatures of two early-type subdwarfs and the low density of interstellar gas near the sun. Some of this data was obtained during the Apollo-Soyuz experiment. Other advances in stellar astronomy center around the role of line blanketing in accounting for the anomalous properties of certain classes of stars and the discovery of the nearly constant luminosity of nova FH Serpentis during the first 60 days. The studies of mass loss and properties of extended stellar atmospheres have received attention and fundamental data on photospheric abund ances have been obtained.

Some planetary nebulae have been observed by Orion- 2 and by sounding rocket observations and will undoubtedly be a field of extended research in the future. Observations of extragalactic nebulae have provided ultraviolet surface brightness maps for a few of the nearer systems while the integrated spectral energy distribution has been measured for a number of more distant systems. These data are exceedingly important for their evolutionary and cosmological implications and suggest an important direction for future space astronomy efforts. While some important data on solar system objects have been obtained by satellite observations the greatest strides in planetary studies have been made by probes and in situ measurements. Satellite observations now and in the near future will probably make their most significant contributions by providing synoptic data on the planets.

The above remarks have been intended to be a brief summary of some of the scientific highlights of the past three years from observations above the Earth's atmosphere. Some of the details and references are contained in the following reviews or in the reports of other Commissions. I would like now to comment on some of the unique contributions that Commission 44 can make to the astronomical community. One obvious role is to act as an agent for coordinating observations of various space craft and ground based facilities. A coordinated campaign to observe $\mathrm{X}$-ray binaries, during the last two years, has been sponsored jointly by Commission 42 and Commission 44 with Y. Kondo acting as its coordinator. An outgrowth of this campaign has been a productive 'Symposium on X-Ray Binaries' held at the Goddard Space Flight Center on 20-22 October 1975 involving some 150 participants. The mechanism of coordination was primarily through the use of Circular Letters. It is difficult to coordinate such diverse observations given the operational constraints of space crafts, the vagaries of weather, and the various observational priorities. The participants in this campaign are to be congratulated for their success and dedication. It is, I think, important for the Commission to be responsive to these needs for coordinated campaigns simply because of uniqueness of space borne astronomical instrumentation. As the user group for satellite observatories has grown and the desire to obtain space observations has increased several other problems have emerged that might be addressed by Commission 44 . The dissemination of information on current observational opportunities is not uniform. To those astronomers not intimately involved in space flight programs it is not always easy to know what instrumentation is currently operational, what opportunities exist for participating as a guest investigator, who should be contacted or what time deadlines exist for responding to announcements of flight opportunities. It may be well for Commission 44 to consider the possibility of a periodic newsletter providing such information. Another problem that has occurred and is worth addressing relates to those astronomers outside the ten member nations of ESA or the USA who may wish to participate as a guest investigator. In general travel assistance is not available to these astronomers from NASA or the ESA nations. While Commission 38 on Exchange of Astronomers provides travel grants for young astronomers wishing to carry out research studies elsewhere, the present rules require an extended visit in excess of six months. Commission 44 might assess the demand for travel support to carrry out satellite observations and investigate mechanisms for filling this need if required. Finally the Commission can play a role in disseminating information, to IAU members, on the new large space programs now in the planning stage and largely international in scope. To this end a meeting session has been scheduled for the current General Assembly.

Among the future programs currently under way or in the planning stage are the IUE (International Ultraviolet Explorer) which is a joint effort of NASA, the U.K. Science Research Council and ESA. The IUE is a $45 \mathrm{~cm}$ Ritchey-Chrétien telescope and an echelle spectrograph, the stellar spectrum being recorded on an SEC Vidicon prior to transmission to the ground. It 
promises to extend high-resolution and relatively low resolution ultraviolet spectroscopy to fainter stars and to galaxies and quasars. The next quantum jump in X-ray and $\gamma$-ray instrumentation should be provided by the HEAO (High Energy Astronomical Observatory) series of space craft, while all areas of astronomy promise to benefit from the Spacelab program. In all the discussion above the emphasis has been upon exploiting the absorption free aspect of observations above the Earth's atmosphere. The possibility of obtaining high spatial resolution and faint limiting magnitudes from space because of the absence of atmospheric image degradation has not as yet become routine. It is technically more difficult and represents the wave of the future. Studies by NASA and ESA for the LST (Large Space Telescope) are well advanced and international efforts to adapt to shuttle astronomy are being pursued with the Spacelab program. There is little question that when Spacelab and LST come to pass a whole new exciting window on the structure of the universe will be opened.

\section{REPORT ON SOLAR OBSERVATIONS FROM SPACE}

(R. M. Bonnet)

The past three years have seen a considerable amount of activity in the area of solar observations from space.

Many countries in the world are still actively engaged in this type of research and participate either in rocket and balloon-born experiments or in more extensive satellite programs. The period extending from 1973 up to 1976 has been characterized by three major events: the launches of Skylab, Salyut 4, and finally OSO-8.

\section{A. Skylab}

Skylab was launched on 1973, May 14. With its set of six instruments built by the Harvard College Observatory, the Naval Research Laboratory, the High Altitude Observatory, American Science and Engineering and the Marshall Space Flight Center, the Apollo Telescope Mount certainly represents the best coordinated approach ever achieved towards high resolution observations from Space over a broad spectral range. It is unlikely that it will be equaled in complexity before the bigger instruments presently designed for the 1980's actually fly on board the shuttle.

(1) The Harvard instrument, during its nine months of operation acquired thousands of EUV spectral scans at $1.3 \AA$ resolution and an even larger number of raster scans with 5 " resolution in up to seven wavelengths simultaneously (1). The continuing analysis of these data has covered EUV spectra of Sunspots (2), structure of active regions (3), loops, limb brightening and spicule analysis, studies of the chromosphere network (4), coronal holes (5) and EUV bright points (6). Significant work is also being done on prominences (7) and their interface to the Corona, currents in active regions, and non-periodic pulsations in EUV emission lines in the quiet Sun $(8,9)$. Diagnostic techniques are being tested to allow inference of plasma parameters from observed line intensities (10).

(2) The NRL was responsible for the chromospheric XUV spectrograph and EUV spectroheliograph. The first instrument recorded 6400 photographic spectra of numerous solar features between $940 \AA$ and $3970 \AA$ with a spectral resolution of $0.06 \AA$, a slit of $2 \times 60^{\prime \prime}$ with no spatial resolution along the slit (11): center-to-limb variation off-limb spectra, plages, flares (12), coronal holes, filaments, prominences, etc. (13).

(3) The Second NRL Instrument recorded 1000 spectroheliograms (14) using an objective grating operating between $180-630 \AA$ giving an excellent spatial resolution of $2^{\prime \prime}$. Among other very impressive observations are those of eruptive prominences (15), the newly discovered macrospicules (16), (17) and numerous observations of flares (18).

(4) The corona could be observed above the disk with the high resolution grazing incidence 
X-ray spectrographic telescope built by ASE. Very high quality pictures were recorded on film in spectral band of $60 \AA$ with an angular resolution better than $2^{\prime \prime}$. In this energy range the solar emission is mostly due to the continuum and lines emitted by the highly ionized constituents of the hot coronal plasma. On the high quality pictures the corona appears highly structured and the structures appear to be the result of dispersed active region magnetic fields (19), (20). Numerous 'bright points' are uniformly distributed across the solar surface (21). Their life-time is approximately 8 hours and proportional to their maximum area which is $\simeq 10^{7} \mathrm{~km}^{2}$. More than 1500 bright points seem to emerge per day.

(5) Another X-ray Telescope placed under the responsibility of the Marshall Space Flight Center operating in five wavelength bands from 5 to $33 \AA$, obtained over $27000 X$-ray photographs of the corona with a resolution better than $2^{\prime \prime}$ and over $1100 \mathrm{~h}$ of X-ray spectral data from 2.5 to $20 \AA$. This highly successful instrument provided new and high quality results on the solar chromosphere, solar flares, (22) and active regions (23).

(6) The corona at distances from the disk between $1.5 R_{\odot}$ and $6 R_{\odot}$ was continuously observed with the HAO white light coronagraph (24). The three-dimensional extent of coronal forms was determined. It was clearly demonstrated with this instrument that there are several time scales on which visual changes in coronal structures occur, ranging from approximately one half rotation to less than hours. These last 'coronal transients' were unexpected: they cause major reorientations of coronal structures by their passage through the coronal medium.

These observations refined those made with a smaller NRL instrument on board OSO-7 (25) which operated until March 1974 and provided several daily recordings of the white light corona from 3 to $10 R_{\odot}(26)$.

In conclusion, many of the efforts described in the framework of the ATM are based on phenomena either newly discovered or newly accessible to study in the Skylab data, and many are in collaboration with the different Skylab experiment teams and with other institutions (27) in particular in the U.K. (28).

\section{B. Salyut 4}

The solar assembly OST on board the Soviet station Salyut 4 has been made at the Crimean Astrophysical Observatory. The telescope has a main mirror with a diameter of $25 \mathrm{~cm}$ and a focal length of $2.5 \mathrm{~m}$. An original concept allowed the coating of this mirror once in orbit, to correct for degradation of the reflectivity usually noticed in Space. A slit jaw camera was used to position selected areas of the solar disk on the slit of the spectrograph, which used crossed dispersion gratings. The regions selected by the astronauts were mostly active regions, flares, prominences and the quiet sun.

The spectral region covered with this instrument is $900-1400 \AA$. The dispersion is $16 \AA \mathrm{mm}^{-1}$ and the spectral resolution $0.4 \AA$. Angular resolution on the image is $5^{\prime \prime}$ along the $3^{\prime}$ long slit, including aberrations plus pointing stability (realized by the astronauts).

More than 200 ultraviolet stigmatic spectra of flocculae, subflares and prominences were obtained in 1975 during two missions, by astronauts Grechko and Gubarev and Klymuk and Swastyanov.

All the lines over the quiet sun are strengthened $3-4$ to $\simeq 10$ times in the spectra of flocculae and subflares, especially those of Nitrogen and Silicon. The most remarkable is the appearance of forbidden lines of Fe XII, $\lambda 1241.7 \AA$ and $\lambda 1349.1 \AA$ usually seen near the solar limb. In some flocculae and subflares, appreciable (reaching $50 \mathrm{~km} \mathrm{~s}^{-1}$ ) line of sight velocities are observed and the Ly- $\alpha$ line is much more widened than in the quiet regions. The appearance of the Fe XII lines and the width of Ly- $\alpha$ point to strong condensations (and possibly to microstructure) of plasma in active formations (29).

\section{C. $O S O-8$}

This NASA spacecraft was launched on 1975, June 21. It contains two major pointed instruments placed under the responsibility of the University of Colorado and the Laboratoire de Physique Stellaire et Planetaire in France. These two pioneering instruments provided for the 
first time high spectral $(0.02 \AA)$ and high spatial (1 to $\left.5^{\prime \prime}\right)$ resolution observations of the chromosphere up to the transition region. The American instrument records profiles of lines ranging from $1200 \AA$ to $2800 \AA$. The French instrument studies the chromopshere in $\mathrm{Ca}^{+} \mathrm{H}$ and $\mathrm{K}, \mathrm{Mg}^{+} \mathrm{H}$ and $\mathrm{K}, \mathrm{Ly}-\alpha$ and $\mathrm{Ly}-\beta$ simultaneously and has an internal rastering capability which allows the analysis of areas of one arc minute maximum with a spatial resolution of 1 " $(30)$. The harvest of results from both instruments is already very impressive and is likely to improve our understanding of the heating mechanism(s) of the chromosphere and corona. For the first time, velocity oscillations have been measured in the chromosphere and their correlations with intensity oscillations studied (31), (32), (33). Numerous bright points and transient phenomena in the chromosphere and the transition region have been observed. High resolution profiles show strong fluctuations on the $1^{\prime \prime}$ scale in the chromospheric network, plages and active regions, coronal holes, prominences and sunspots (34), (35). The simultaneity of observations at different levels in the atmosphere which is achievable with the French instrument allows the study of the vertical extension of the spot structure and little correlations have been found so far between images taken in O VI $(1032 \AA)$ and in white light. Magnetic field restructuring has been observed on 1975, August 6, on active region no. 617/618 (36). Non-thermal broadening of transition region lines are being observed and will provide useful information as to the propagation of waves in the upper solar atmosphere. Both instruments are operated from LASP on the campus of the Colorado University and more than twenty guest investigators coming from the U.S., Europe and U.S.S.R. participate in the operations and in the data analysis.

The successful operations of the two pointed instruments on OSO-8 will undoubtedly provide a high scientific return. In addition two other experiments located in the spinning wheel of the spacecraft study the X-ray emission of the Corona:

(1) A mapping X-ray heliometer under the responsibility of Lockheed Research Laboratory operating between 2 and $30 \mathrm{keV}$ with an angular resolution of $2^{\prime}$.

(2) An X-ray crystal spectrometer and polarimeter under the responsibility of Columbia University operating between 2 and $8 \mathrm{keV}$.

\section{Other Solar Satellites}

Apart from the 3 major events described above, several less extensive but highly successful and important observations have been conducted with other satellites, rockets or balloons all over the world.

The French D2B satellite launched on 1975, September 27 carries a small grazing incidence spectroheliometer. This instrument takes low resolution spectra of the sun between $170 \AA$ and $1250 \AA$ and makes spectroheliograms in 7 different lines corresponding to highly ionized atoms such as Fe XV and Fe XVI. A continuous monitoring of the solar flux is underway with this instrument which has been very carefully calibrated and measures the absolute flux of the Sun with an accuracy varying between $10 \%$ and $20 \%$.

A solar X-ray $(1-2 \AA)$ and Ly- $\alpha$ flare monitor was placed into orbit on 1975 , February 24 by the third Japanese scientific satellite 'Taiyo' (37).

In Europe, observations made with the Utrecht Solar hard X-ray spectrometer on board the ESRO TD 1-A satellite have shown that solar X-ray bursts usually consist of many short lived discrete bursts with rise and decay times of less than five seconds (38).

In U.S.S.R., the highly successful Intercosmos program has continued. New satellite lines close to the $\mathrm{Mg}$ XII line at $8.42 \AA$ were observed on board 'Intercosmos 4, 7' in the spectra of flares during the starting phase (39) with a low level of polarization by the scientists at the Physical Lebedev Institute in Moscow (40).

High resolution profiles of this same line were recorded from 'Intercosmos 7' and allowed the investigation of the thermal structure of the hot cores of coronal condensations and their changes from day to day (41).

India recently joined the community of Solar Space Experimenters with the launch on 1975, April 19 by a Soviet rocket of the first Indian satellite 'Aryabhatta'. The satellite carries several instruments one of which monitors the solar neutrons and gamma rays. The experiment aims at detecting the possible impulsive emission of energetic neutrons in the 10 to $500 \mathrm{MeV}$ region 
and gamma rays in the $200 \mathrm{keV}$ to $20 \mathrm{MeV}$ energy region at times of violent solar activity as well as during quiet conditions on the surface of the Sun. Such observations are expected to give important information on particle acceleration, the solar atmosphere and nuclear processes that take place at times of solar flares (42).

Helios I, which was launched successfully on 1974, December 10 measured solar cosmic rays

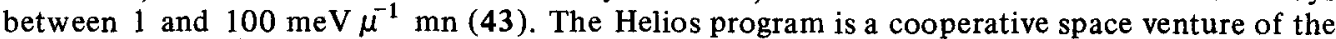
German Federal Republic and the United States.

In cooperation with the Soviets, the Stereo-I mission which has been carried out under the responsibility of the Groupe de Radioastronomie Spatiale, Meudon, has given simultaneous observations of type I and III bursts at $169 \mathrm{MHz}$ between the Soviet probe Mars-3 and Nancay (44). The time profile of type III bursts (45) has been found to be independent of the direction of observation. In all cases investigated, the directivity of bursts is larger than computed from an isotropic point source radiating in a spherical corona with isotropic inhomogeneities. The directivity of type I bursts has also been deduced: the observations can be quantitatively interpreted assuming that type I radiate into a randomly oriented beam pattern of constant shape (46).

\section{E. Rockets and Balloons}

Most rockets launched so far carried sophisticated instruments often allowing high spatial or/and high spectral observations. Most impressive of all seems to be the new stigmatic EUV spectrograph of NRL launched on 1975 , July 21 covering the spectrum from $1175 \AA$ to $1700 \AA$ with a spectral resolution of $0.06 \AA$ with the capability of being positioned from the ground on selected regions such as plages and sunspots. The spatial resolution of 1 " allows the observation of the small scale variations of the continuum intensity and temperature differences of $500 \mathrm{~K}$ at temperature minimum. Highly resolved profiles of transition region zone lines in sunspots indicate downward motions in a very small area of the umbra.

Several efforts have been spent to study the line shape of the $584 \AA$ and $304 \AA$ lines of He I and He II (47). A joint French-American payload was successfully launched by a rocket of the University of Colorado on 1975, January 20. The French instrument carried an absorption cell filled with helium which measured a FWHM of the $584 \AA$ line equal to $130 \mathrm{~m} \AA$ (total Sun) (48), (49), (50).

Observations in the XUV region of the spectrum have been conducted actively in the U.S. (NRL: Spectroheliograms with $4^{\prime \prime}$ resolution between $200 \AA$ and $630 \AA$ ) and U.K. (Leicester University: Bragg Crystal Spectrometers covering between $4 \AA$ to $23 \AA$ ).

Several groups have been involved in the measurements of the absolute and relative intensity (center-to-limb) of the solar disk. The Harvard College Observatory completed several measurements of the quiet sun absolute intensity with a spectral resolution of $0.03 \AA$ between $1174 \AA$ and $3200 \AA$ with moderate spatial resolution (51), (52). The uncertainties are believed to be less than $\pm 15 \%$. Absolute intensity and flux measurements were also undertaken at NRL $(0.06 \AA)$ LASP and AFCRL $(\Delta \lambda=2-10 \AA)$.

Other elaborate instruments were launched in Japan (53) and France (54) and measured the temperature structure comparing center-to-limb intensities. The French measurements allow the determination of continuum source-functions and opacities. As a result, the brightness temperature is now found to be very close to $4400 \mathrm{~K}$ exceeding by $300 \mathrm{~K}$ that found at $200 \mu \mathrm{m}$ in the far infrared, thereby indicating fairly large departures from LTE in the metals responsible for the emission in the UV continuum (55).

The Appleton Astrophysics Research Unit Division at Culham extended the temperature determinations higher in the atmosphere by measuring the ratio of spectral intensities on the disk and just above the limb (56). High resolution spectra also obtained at Culham provide good evidence for non-thermal mechanical broadening of optically thin lines (57).

The Lockheed Palo Alto Research Laboratory has been very active in the past years concentrating its rocket program on spectroscopic studies of the solar corona at X-ray wavelengths (58) and Solar Flares (59).

Balloons were also used in the past years mostly in the far infrared and in the visible. High 
accuracy photometry was performed at the Geneva Observatory between $80 \mu \mathrm{m}$ and $600 \mu \mathrm{m}$ indicating a flat temperature minimum of $4100 \mathrm{~K}$ between $120 \mu \mathrm{m}$ to $200 \mu \mathrm{m}(60)$. The fine structure of the Solar Atmosphere in the visible was studied in Japan (61) and with the Spectro-Stratoscope in Germany. This last instrument allowed the study of the temporal evolution in the granulation as well as its center-to-limb variation.

Continuing data analysis on past satellites and rocket experiments have also greatly contributed to improving our knowledge of the physical processes occurring in the Sun. The highly successful NASA Spacecraft OSO-5 was reactivated in 1975 , providing nearly continuous monitoring of the Solar Activity in both X-rays (UCL) and Ly- $\alpha$ (Paris University).

\section{F. Conclusion}

This report is certainly not complete. It has been written on the basis of information either kindly sent to the author by several investigators all around the world, or available in the literature. It shows that solar physics investigations from space are entering a new era with bigger and more complex satellite and rocket instruments. These instruments open the way to the even more complex assemblies and clusters of telescopes which are envisaged to fly on board the space shuttle in the 1980's. It is expected that the intermediate period extending between now and the 1980's will be fruitfully used to prepare the new era from the point of view of generating both powerful instruments and exciting programmes.

\section{REFERENCES}

1. Reeves, E. M.et al.: 1975,Proc. AIAA/AGU Conf. Scient. Exper. Skylab (in press).

2. Foukal, P. V.et al.: 1974, Astrophys. J. Letters 193, L143.

3. Noyes, R. W.et al.: 1975, IA U Symp., 68, 3.

4. Reeves, E. M.et al.: 1974, Astrophys. J. Letters 188, L27.

5. Huber, M.C. E. et al.: 1974, Astrophys. J. Letters 194, L115.

6. Timothy, J. G. et al.: 1975, Proc. XVII COSPAR Meeting (in press).

7. Schmahl, E. J.et al.: 1974, Solar Phys. 39, 337.

8. Vernazza, J. E. et al.: 1975, Astrophys. J. Letters 199, L123.

9. Withbroe, G. L. et al.: 1975, Astrophys. J. (in press).

10. Reeves, E. M. and Dupree, A. K. 1975, Proc. Fourth Int. Conf. on Beamfoil Spectroscopy (in press).

11. Tousey, R. et al.: 1974, IAU Symp. 57, 491.

12. Scherrer, V. E. and Tousey, R. 1975, Proc. Int. Conf. on 'X rays in Space' 2, 986, Univ. of Calgary.

13. Feldman, U. et al.: 1975, Astrophys. J. Letters 199, L67.

14. Tousey, R. et al.: 1973, Solar Phys. 33,265.

15. Tousey, R. 1975, in 'A Discussion on the Physics of the Solar Atmosphere', Phil. Trans. Roy. Soc. London (in press).

16. Bohlin, J. D. et al.: 1975, Astrophys. J. Letters 197, L133.

17. Moe, O. K., Engvold, E. and Beckers, J.: 1975, Solar Phys. 40, 65.

18. Scherrer, V. E. and Tou sey, R.: Proc. AIAA/AGU Conf. Scient. Exper. Skylab (in press).

19. Vaiana, G. S. et al.: 1973, Astrophys. J. Letters 185, L47.

20. Vaiana, G. S., Krieger, A. S. and Timothy, A. F.: 1973, Solar Phys. 32, 81.

21. Golub, L. et al.: 1974, Astrophys. J. Letters 189, L93.

22. Marshall Space Flight Center Skylab Apollo Telescope Mount Experiment Systems Mission Evaluation, NASA Techn. Mem. N 74-28310, June 1974.

23. Smith, J. B. Jr. et al.: 1975, XVIII COSPAR Meeting, Abstract III B-2-1.

24. MacQueen, R. M. et al.: 1974, Astrophys. J. Letters 187, L85.

25. Koomen, M. J. et al.: 1975, Appl. Opt. 14, 743.

26. Stewart, R. T. et al:: 1974, Solar Phys. 36, 219.

27. Pye, J. P. et al.: 1974, IAU Symp. 68.

28. 'Solar Physics in the United Kingdom: Solar Physics Study Panel Subcommittee on Planetary Sciences', British Nat. Comm. Space Research, September 1975.

29. Bruno, A. V. et al.: 1975, XVIII COSPAR Meeting, Abstract IIIB-2-6.

30. Artzner, G. E. et al.: 1975, submitted to Space Sci. Instr.

31. Vial, J.C. et al.: 1975, 'Periodic Temporal Phenomena in the Solar Chromosphere as Observed from OSO-8', 147 Meeting of AAS. 
32. Chipman, E. G. et al.: 1975, 'Velocities in the Solar Chromosphere Observed in the Si II $\lambda 1816$ Line,' 147 Meeting of the AAS.

33. Lites, B. W. et al.: 1975, 'Persistent Velocities in the Middle Chromosphere,' 147 Meeting of the AAS.

34. Bonnet, R.M. et al.: 1975, 'Spatial Variations of Solar Profiles of $\mathrm{Ca}+\mathrm{H}$ and K, $\mathrm{Mg}+\mathrm{h}$ and k, H I Ly- $\alpha$ and Ly- $\beta$ Lines as Observed from OSO-8,' 147 Meeting of the AAS.

35. Bruner, E.C. Jr. et al.: 1975, 'High Resolution Spectroscopy from Orbiting Solar Observatory VIII: Transition Zone Dynamics Over a Sunspot,' 147 Meeting of the AAS.

36. Artzner, G. E. et al.: 1975, 'Evolution of Sunspot and Active Region Morphology as Observed on the Resonance Lines of $\mathrm{Ca}+(\mathrm{H} . \mathrm{K}) \mathrm{Mg}+(\mathrm{k}, \mathrm{h}) \mathrm{H} \mathrm{I}(\mathrm{Ly}-\alpha, \mathrm{Ly}-\beta)$ and $\mathrm{O} \mathrm{VI}$ from OSO-8,' 147 Meeting of the $A A S$.

37. Hirao, K.: 1975, Rept. Ionosph. Space Res. Japan 29.

38. de Feiter, L. D., de Jager, C. and Beck, H. V. Van: 1975, XVIII COSPAR Meeting, Abstract III, B-2-10.

39. Vaienstein, L. A. et al.: 1975, XVIII COSPAR Meeting, Abstract III-B-2-7.

40. Tindo, I. P. et al :: 1975, XVIII COSPAR Meeting, Abstract III-B-2-9, No. 64, submitted to Solar Phys.

41. Jakimiec, J. et al :: 1975, XVIII COSPAR Meeting, Abstract III-B-2-8.

42. Shirke, J. S.: 1975, Physics News, Bull. Indian Phys. Assoc. 6, No. $2,48$.

43. Kurrow, H. et al:: 1975, XVIII COSPAR Meeting, Abstract No. II-1-6a.

44. Caroubalos, C. and Steinberg, J. L.: 1974, Astron. Astrophys. 32, 245.

45. Caroubalos, C., Poquérusse, M. and Steinberg, J. L.: 1974, Astron. Astrophys. 32, 255.

46. Steinberg, J. L., Caroubalos, C. and Bougeret, J. L.: 1974, Astron. Astrophys. 37, 109.

47. Doschek, G. A., Behring, W. E. and Feld man, U.: 1974, Astrophys. J. Letters 190, L141.

48. Delaboudinière, J. P. and Carabetian, C.: 1975, Space Sci. Instr. 1, 91.

49. Delaboudinière, J. P. and Crifo, J. R.: 1975, XVIII COSPAR Meeting, Abstract No. III-B-2-4.

50. Rense, W. A. and Cushman, G. W.: 1975, XVIII COSPAR Meeting, Abstract No. III-B-2-3.

51. Kohl, J. L. and Parkinson, W. H.: Astrophys. J. (in press).

52. Kohl, J. L. and Parkinson, W. H.: 1975, Bull. Am. Astron. Soc. 7, 450.

53. Nishi, K.: 1975, Solar Phys. 42, 37.

54. Samain, D. et al.: 1975, Astron. Astrophys. 39, 71

55. Vernazza, J.E. et al .: 1975, Astrophys. J. Suppl. in press.

56. Firth, J. G. et al.: 1974, Monthly Notices Roy. Astron. Soc. 166, 543.

57. Boland, B. C. et al.: 1975, Monthly Notices Roy. Astron. Soc. 171, 697.

58. Acton, L. W. and Catura, R. C.: 1975, Proc. Roy. Soc. Meeting on the Physics of the Solar Atmosphere.

59. Zaumen, W. T. and Acton, L. W.: 1974, Solar Phys. 36, 139.

60. Stellar, P. et al.: 1975, Solar Phys. 40, 337.

61. Hirayama, T. et al.: 1974, Bull. Inst. Space Aeronaut. Sci, Univ. Tokyo, 10, No. 1, B, 183 (in Japanese).

\section{ULTRAVIOLET ASTRONOMY (A. D. Code)}

In the period since the last IAU report a wealth of observational material on the ultraviolet spectra of celestial objects has been obtained. The report of Commission 29 on Stellar Spectra includes a section on 'Ultraviolet Stellar Observations'. In that discussion many of the scientific results on stellar objects obtained with Copernicus, with the TD-1A S/59 and S2/68 experiments, and with OAO-2 are presented. Additional material on results with Skylab-S183, AURA, ANS, Mariner 9 and Manned space craft and rocket balloon data are discussed. In this account we shall attempt to supplement rather than duplicate the material presented in the Commission 29 report. For a more complete account the reader is invited to review both reports.

Absolute calibration of the energy distribution of stars has been carried out by a number of investigators. The agreement between these independent determinations represents a substantial improvement over past determinations. Bohlin, Frimout and Lillie (1974) at the University of Colorado carried out a calibration in the wavelength region from $1700 \AA$ to $3300 \AA$. This calibration was based primarily on National Bureau and Standards calibrated tungsten ribbon filament lamps and cesium telluride photodiodes which were used to calibrate standard photomultipliers. The molecular branching ratio technique confirmed the general shape of their calibration curve. Two independent calibrations of the TD-1A S2/68 spectrometer were performed at Liege and Edinburgh (Humphries et al. 1974). The basic standard for these calibrations were black body sensors and each of these investigations were carried out carefully 
and independently. The Liege and Edinburgh calibrations agreed with each other to within $20 \%$. The calibration adopted for TD-1 is the mean of the Liege and Edinburgh calibrations. At Johns Hopkins University a calibration extending from $1180 \AA$ to $1680 \AA$ has been published (Henry et al., 1975). The stellar data were obtained with a flight instrument carried on Apollo 17. The calibration was based upon comparison with reference photomultipliers which were calibrated against National Bureau of Standards photodiodes. A calibration between $1300 \AA$ and $3000 \AA$ was made by Bless, Code and Fairchild $(1974 ; 1976)$ using radiation from the University of Wisconsin Physical Science Laboratory synchrotron. The synchrotron source is exceptionally well suited to the problem. Not only is the spectrum of such radiation accurately predicted by theory, but the absolute value of the radiation can be determined simply by counting the electrons circulating in the storage ring. The agreement between these different calibrations is quite good longward of $2500 \AA$. Shortward of $2500 \AA$ individual calibrations do not differ anywhere by more than $30 \%$. This general agreement between recent calibrations is encouraging in view of the large discrepancies that formerly existed in the ultraviolet. The fact that they are based on completely different techniques employing thermal sources, non-thermal sources, or black body sources calibrated in the visual, increases the confidence in the general flux levels in the vacuum ultraviolet. Absolute calibration efforts are continuing at several institutions.

The absolute calibration of stellar fluxes has led to significant improvements in our knowledge of fundamental parameters of stars. Since for stars with effective temperatures greater than $10000 \mathrm{~K}$ more than $70 \%$ of the energy is radiated shortward of $3000 \AA$ it is clear that ultraviolet data are basic to an understanding of early-type stars. A number of investigations have been carried out on effective temperature scales and bolometric corrections. Beeckmans et al. (1974) obtained semi-empirical bolometric corrections by fitting blanketed model atmosphere to the UV spectrophotometry given by the TD $1 \mathrm{~S} 2 / 68$ scanning spectrometer. Heintze, Kamperman, Sakhibulin (1975) were able to estimate effective temperatures of main sequence stars observed by S59 by a photometric reduction of the UV spectra of these stars. Nandy and Schmidt (1975) obtained temperature scales from comparing UV fluxes with those computed from a grid of model atmospheres. Code et al. $(1974 ; 1976)$ have determined empirical bolometric corrections and effective temperatures for 32 stars with angular diameters measured with the Narrabri stellar intensity interferometer. The agreement between the derived stellar surface fluxes and blanketed model atmospheres is excellent for stars with effective temperatures less than $30000 \mathrm{~K}$ where plane parallel atmospheres are valid. For hotter main sequence stars and supergiants, atmospheric extension introduces an ambiguity in the concept of effective temperature (de Jager, 1975). The observations are consistent, however, with the results for extended model atmospheres (Cassinelli and Hartmann, 1975). For those stars with observed energy curves, angular diameters, and reliable trigonometric parallaxes, Code et al. (1976) have constructed an empirical fundamental HR diagram, $\log L$ vs $\log T_{\mathrm{e}}$. Code (1975) has extended the determination of empirical bolometric corrections of early-type stars to a larger sample of stars and presented a preliminary mean bolometric correction and temperature scale for main sequence stars.

The spectral distribution of hot subdwarfs have been studied by several investigators. Holm (1975) has discussed the OAO-2 observations of sixteen early-type subluminous stars. The spectral distribution of stars like $\mathrm{BD}+284211$ and Feige 24 are consistent with effective temperatures in excess of $60000 \mathrm{~K}$. Because of the low luminosity and high surface gravity of these stars plane parallel LTE model atmospheres show good agreement with the observations. C. C. Wu is undertaking a study of a larger body of data on hot subdwarfs obtained with ANS. Carnochan et al. (1975) have conducted a sky search for ultraviolet objects using data from the S2/68 sky survey telescope on TD-1A. They conclude that the frequency population of hot objects lying a few magnitudes below the main sequence is probably much greater than had been thought hither to. Henry et al. (1976) have attempted to place limits on the space density of $O$ subdwarfs and hot white dwarfs based on EUV surveys. Their upper limits are about a factor of 10 higher than what is estimated on theoretical grounds, but not inconsistent with the results of Carnochan et al. Mewe et al. (1976) have reported soft X-rays from $\alpha$ Aur and $\alpha$ CMa B. Observations at longer wavelengths in the EUV have been reported for several stars. Lampton et 
al. (1976) carried out a survey on the Apollo--Soyuz mission. A 37-cm diameter grazing incidence mirror assembly with a field of view of 2.5 or 4.3 deg illuminated a pair of channel electron multiplier photon detectors through filters which isolated three bands: $170-620 \AA$, $114-150 \AA$ and $55-150 \AA$. The DAwk star $\mathrm{HZ} 43$ was observed in all channels and is consistent with a black body temperature of $110000 \mathrm{~K}$. It is reported that EUV flux was also observed for Feige 24 and for proxima Centauri. These observations confirm the high temperatures assigned to hot subdwarfs and also indicate that the interstellar column density is very low in the direction of these objects.

Variable star observation obtained with the OAO-2 space craft have been summarized by Holm (1975). These include observations of 15 eclipsing systems observed throughout most of their light variation except for the long period systems 31 Cygni and 32 Cygni. Among the intrinsic variables are 5 classical cepheids, RR Lyrae, $7 \delta$ Scuti variables, $9 \beta$ Cephei variables, $7 \alpha \mathrm{CVn}$ type, 4 nova or nova-like variables, the supernova of 1972 in NGC 5253 and a number of miscellaneous variables. A discussion of the ultraviolet light curves of cepheids has been given by Hutchinson (1975). Lesh has studied the OAO photometry and spectral scans of $\beta$ Cephei stars. Beeckmans et al. (1976) have analyzed TD-1A S59 and S2/68 data on these type of variables. The initial analysis of the 1972 supernova NGC 5253 was made by Holm, Wu and Caldwell (1974). Unlike the previously reported behavior of the nova FH Ser: (Gallagher and Code, 1974), all of the supernova light curves decline simultaneously with the possible exception of a rise in the $2460 \AA$ curve some 60 days after maximum. The measurements of the peculiar A type spectrum variables is particularly instructive. Studies primarily by Molnar (1973a; $1973 \mathrm{~b}$ ) of the systems $\alpha^{2} \mathrm{CVn}$ and $\epsilon$ UMa are interpreted as a variation in line blanketing that reduces the ultraviolet flux and enhances the visual flux. Investigations of line blocking have been carried out with TD-1A S/59 data. Underhill and van der Hucht (1975) measured the line blocking per $10 \AA$ in the observed wavelength bands for $35 \mathrm{~B} 6$ to $\mathrm{A0}$ stars, and discussed the differences between peculiar and normal stars. Investigations of interstellar extinction based upon TD-1 A data have been published by Nandy et al. (1975). The $2200-\AA$ feature was found in all reddened stars investigated. Moreover the $2200-\AA$ bump was found to be remarkably similar in all stars. These conclusions are in agreement with those of Savage (1975) based on further studies of OAO-2 data. The apparent absence of a $2200-\AA$ bump from analysis of Celescope data (Davis, 1975) has now been resolved as being due to a systematic error in the early Celescope magnitudes. Variations in the ultraviolet extinction curve have been investigated by Viotti and Lamers (1975).

Sky surveys in the ultraviolet have been conducted by several groups. Objective prism spectra have been obtained from Gemini 11 (Morgan, et al., 1975) and Skylab by Henize et al. (1975) and by Courtés et al. (1975) with the French S183 Skylab experiments. The French group has also carried out very wide field investigations with thee UV 'Janus' camera utilizing a sounding rocket. Preliminary results from S183 yield photometry at $2574 \AA$ on more than 3000 stars to a limiting visual magnitude 12 .

A variety of observations of extragalactic nebulae are now available. Ultraviolet photographs at $2500 \AA$ were obtained with the S183 Skylab camera which show only the Population I components. Evans et al. (1975) have obtained ultraviolet surface brightness measurements in the Large Magellanic Cloud with the TD-1 A S2/68. Borgman et al. (1975) have also measured regions in the LMC near 30 Doradus with ANS. In both of these observations no indication of the $2200 \AA$ feature is found. A number of observations of M 31 and other galaxies have been made with TD-1A and ANS. Code and Welch (1975) have investigated early type galaxies observed by OAO-2. They find that the radio bright elliptical M 87 is brighter in the ultraviolet than other non-radio $\mathrm{E}$ and SO galaxies. This may be due to the burst of star formation accompanying the active nuclear events in radio galaxies proposed by Van den Bergh (1975). If so it is a feature that must be taken into account in cosmological investigations of large red shift galaxy clusters.

In closing this section it is appropriate to remark that this survey omits many of the fascinating results currently being pursued in ultra-violet astronomy. 


\section{REFERENCES}

Beeckmans, F.etal.: 1976, IAU Coll. 29.

Beeckmans, F. et al.: 1974, Astron. Astrophys. 33, 93.

Bless, R. C. et al.: 1974, Wisconsin Astrophys. No. 1; 1976, Astrophys. 203.

Bohlin, R. C. et al :: 1974, Astron. Astrophys. 30, 127.

Borgman, J. et al.: 1975, Astron. Astrophys. 40, 461.

Carnochan, D. J. et al.: 1975, Phil. Trans. Roy. Soc. London A 279, 479.

Cassinelli, J. P. and Hartmann, L.: 1975, Wisconsin Astrophys. No. 14.

Code, A. D.: 1975, Dudley Obs. Rep. No. 9.

Code, A. D. et al.: 1974, Wisconsin Astrophys. No. 2; 1976, Astrophys. J. 203.

Code, A. D. and Welch, G. A.: 1976, Bull. Am. Astron. Soc.

Courtes, G. et al.: 1975, Phil. Trans. Roy. Soc. London A 279, 401.

Davis, R. J.: 1975, Phil. Trans. Roy. Soc. London A 279, 345.

De Jager, C.: 1975, Phil. Trans. Roy. Soc. London A 279, 421.

Evans, R. G. et al.: 1975, Phil. Trans. Roy. Soc. London A 279, 473.

Gallagher, J. S. and Code, A. D.: 1974, Astrophys. J. 189, 303.

Henize, K. G. et al.: 1975, Astrophys. J. 199, L1 19.

Henry, P. et al.: 1976, Astrophys. J. (April 1976).

Henry, R. C. et al.: 1975, Astrophys. J. 201, 613.

Holm, A. V.: 1975, Phil. Trans. Roy. Soc. London A 279, 459.

Holm, A. V. et al.: 1974, Publ. Astron. Soc. Pacific 86, 296.

Humphries, C. M.et al.: 1974, Astron. Astrophys. (submitted).

Hutchinson, J. L.: 1975, in Fischel and Sparks (eds.), 'Cepheid Modeling' p. 5, NASA SP-383.

Lampton, M. et al.: 1976, Astrophys. J. Letters 203:

Lesh, J. R.: submit ted to Astrophys. J.

Mewe, K.et al.: 1976, Space Res. XVI.

Molnar, M. R.: 1973, Astrophys. J. 179, 527.

Molnar, M. R.: 1973, Bull. Am. Astron, Soc. 5, 325.

Morgan, T. H. et al.: 1975, Astrophys. J. 197, 371.

Nandy, K. et al.: 1975, Astron. Astrophys. 44, 195.

Savage, B. D.: 1975, Astrophys. J. 199,92.

Underhill, A. B. and Van der Hucht, K. A.: 1975 1976, Astron. Astrophys. (submitted).

Van den Bergh, S.: 1975, Ann. Rev. Astron. Astrophys. 13, 217.

Viotti, R. and Lamers, H. J.: 1975, Astron. Astrophys. 39, 465.

\section{BRIEF REVIEW OF RECENT RESULTS IN X-RAY ASTRONOMY}

\section{(P. Gorenstein)}

Profound changes in the rate of progress in X-ray astronomy began with the advent of the first orbiting X-ray observatory, 'Uhuru', launched 1970, December 12 from the San Marco platform in Kenya. The long time available for observations (years, instead of the few minutes available in rocket flights), and the much finer temporal and spatial resolution, have resulted in a qualitative change in our understanding of the X-ray sky in the 2 to $10 \mathrm{keV}$ range. The increased positional accuracy $\left(\sim 1^{\prime}\right)$ was particularly important because it made possible the identification and study of the optical and radio counterparts of X-ray sources. The Uhuru satellite operated continuously for four years. Other satellite missions, such as OAO-3, OSO-7, ANS (Astronomical Netherlands Satellite), Ariel 5, and SAS-3 followed and most are still in operation. They have extended the Uhuru results to broader domains of time and X-ray energy. Approximately 200 individual new sources have been detected. During this time improved rocket instruments have produced new results on soft X-rays $(0.1-2 \mathrm{keV})$ from individual sources and the soft $\mathrm{X}$-ray background. Fourteen objects which are primarily soft $\mathrm{X}$-ray sources have been detected and it has been shown that the soft X-ray background includes a major new high temperature component of the interstellar medium. With these missions, we have reached a new level in understanding X-ray emission mechanisms and the connection between X-ray sources and many of the astrophysical objects of greatest present interest such as collapsed objects (neutron stars and black holes), quasars and Seyfert galaxies, and clusters of galaxies. 
Soft X-ray results, in particular, have produced new insights into previously known objects such as supernova remnants, cataclysmic variables, flare stars, and stellar coronae and have changed our perception of the interstellar medium.

\section{A. The $X-R a y S k y(2-10 \mathrm{keV})$}

The UHURU Catalog of X-ray Sources (Figure 1,p.210) contains 161 sources and one can claim perhaps 40 additional by including other results especially those on variable sources. The weakest source in the survey corresponds to a flux of about $3 \times 10^{-11} \mathrm{erg} \mathrm{cm}^{-2} \mathrm{~s}^{-1}$ at Earth, the strongest (Sco X-1) to about $3 \times 10^{-7} \mathrm{erg} \mathrm{cm}^{-2} \mathrm{~s}^{-1}$. There have been transient sources which have temporarily exceeded this intensity. The sources divide naturally into two groups: those that are clustered at low latitude along the galactic plane (The Milky Way) and those that spread more uniformly about the sky. Most of the sources we observe along the Milky Way are probably stellar systems in our own Galaxy. Most of those we observe at high galactic latitude (toward the galactic pole) are connected with extragalactic objects.

Studying in detail the distribution of sources in the sky, the relationship between the number of sources and apparent luminosity, the rough spectral information available, and making use of the identifications with visible light objects, we have arrived at the following general conclusions:

(a) The luminosity of the galactic $X$-ray sources is in the range $10^{35-38} \mathrm{erg} \mathrm{s}^{-1}$. X-ray sources are among the most luminous objects in our galaxy and the mechanism for production of X-rays in these objects must be therefore connected with their basic energetics.

(b) The number of sources in our Galaxy in this range of luminosity is of the order of 100. Thus, X-ray stars are only $10^{-9}$ times as abundant as normal stars. (However, if one considers only massive blue supergiant stars and globular clusters then the fractional component of X-ray sources is much higher).

(c) Among the identified sources are supernova remnants, and 6 objects associated with binary stars.

(d) Aside from the supernova remnants, virtually all of the galactic X-ray sources are variable in one time regime or another, frequently in several regimes simultaneously such as the pulsating transient sources studied by Ariel 5 .

(e) All galaxies are X-ray emitters at some level. Normal galaxies (M 31, Large and Small Magellanic Clouds) emit about $10^{39} \mathrm{erg} \mathrm{s}^{-1}$ due to the integrated emission from X-ray stars. In these galaxies $L_{x} / L_{\mathrm{rad}}=10^{-5}$ ( $L_{\boldsymbol{x}}$ is the X-ray and $L_{\mathrm{rad}}$ the visible light luminosity).

(f) In active galaxies, such as Seyferts (NGC 4151), radio galaxies (NGC 5128), or quasars (3C273), the X-ray luminosity is much greater, $10^{42}$ to $10^{46} \mathrm{erg} \mathrm{s}^{-1}$, and also $L_{x} / L_{\mathrm{rad}}$ is much greater. It is likely that the X-rays we observe originate in small regions near the nucleus of the galaxies where the occurrence of explosive events has been inferred from optical and radio studies.

(g) X-ray emission associated with clusters of galaxies appears to originate from extended low density regions between galaxies, as well as from individual galaxies. While there exists no obvious relation between X-ray emission and the extended radio and optical halos observed in clusters, the various emissions occur in overlapping regions of space necessitating a single underlying phenomenon. The luminosity of clusters is of $10^{43}$ to $10^{45} \mathrm{erg} \mathrm{s}^{-1}$. When an active galaxy is present, as in Virgo and Perseus, the X-ray emission appears centered on the active galaxy. At present, we attribute the extended X-ray emission to the existence of a hot intergalactic gas containing approximately the same mass as is present in the condensed galaxies of the cluster.

(h) The diffused X-ray background in the 2 to $10 \mathrm{keV}$ range appears to be isotropic to within small observational limits $(<5 \%)$. This implies an extragalactic origin at large distances. (This is quite different from the soft X-ray background which is decidedly anisotropic and probably of galactic origin.) If due to the contribution of unresolved single sources, these objects must belong to a different class from the extragalactic sources already detected. If due to diffused mechanisms, then its origin must be tied to some aspect of the intergalactic medium. Its study might then have a bearing on a number of outstanding astronomical problems, such as the mass density of the University, early galaxy formation, and galactic activity. 
The most detailed measurements have been carried out upon the class of objects known as binary X-ray sources. For example, X-ray measurements plus observations of the optical counterpart have led to the conclusion that Her X-1 and 3U0900-40 are neutron stars within a binary system and that $\mathrm{Cyg} \mathrm{X-1}$ is probably a black hole. Masses of the compact objects have been estimated which apparently require those conclusions.

\section{B. The Soft X-Ray Sky $(0.1-2 \mathrm{keV})$}

While the results described above were being obtained in the $2-10 \mathrm{keV}$ region by Uhuru and other satellites, significant progress was also being made in the lower energy region by ròckets and instruments aboard the OAO-3, ANS, and SAS-3 satellites. Progress has not been as rapid as at higher energies for several reasons: the interstellar medium is only partially transmitting, the instruments have been rather modest, and the source intensities are lower in this energy regime. Nevertheless, a total of fourteen soft X-ray sources have been identified and important studies have been carried out upon the structure of the soft X-ray background. The identified sources include supernova remnants, a recurrent nova, SS Cygni stars, flare stars, the coronae of nearby stars, white dwarfs, and a soft component of emission from compact binary systems. All of the well established sources are optically identified. Supernova remnants have been the most extensively studied of these soft sources. They are explained as the interaction of a several million degree shockwave with the ambient interstellar medium resulting in thermal emission of $\mathrm{X}$-rays. There is now at least an approximate picture of how the shockwave evolves with time. The morphology of the individual remnants is understood to result from clouds initially present in the interstellar medium reacting to the influence of the shockwave.

Recent results on the soft X-ray background have established that it has a diffuse interstellar origin. The soft X-ray structure of the background have been mapped on a scale on several degrees over a significant fraction of the sky. It appears to be a network of extended regions of enhanced emission highly convoluted with regions of enhanced absorption. Thus, we hypothesize that a new component of the interstellar medium has been identified. This new component has a temperature of $10^{6} \mathrm{~K}$, a particle density in the range $10^{-2}$ to $10^{-3} \mathrm{~cm}^{-3}$ and occupies a substantial volume of the total medium.

\section{Gamma-Ray Bursts}

The discovery of cosmic gamma-ray bursts was announced in 1973 by the Vela satellite group. Photon bursts in the hard X-ray to gamma-ray range were detected simultaneously in several widely separated satellites. The duration of the events varies within the range $0.1-30 \mathrm{~s}$ with no unique time structure. Larger duration events consist of several bursts separated by a few seconds. The high energy cutoff for the bursts is an exponential function with an e-folding energy of about $150 \mathrm{keV}$. The low energy cutoff is variable and is about $20 \mathrm{keV}$. The relative time delay for the same event as observed in different satellites provided sufficient information to place the event at distances beyond the solar system. Other satellite instruments, particularly IMP-6, as well as balloon experiments, searched their data records and were able to provide additional information about the spectra, time structure, and location of the events.

The quest for more information about gamma-ray bursts has blossomed into one of the most exciting aspects of high energy astrophysics. A total of 30 bursts have been catalogued. Position information has been too crude to allow their optical identification. They occur at a rate of about 5 per year with a strength exceeding $10^{-5} \mathrm{erg} \mathrm{cm}^{-2}$. They are widely distributed across the sky and their distribution is consistent with isotropy. It is difficult to construct a precise number intensity distribution curve because of the limited event statistics but $\mathrm{d} N(>s) / \mathrm{d} S$, (the differential number of events exceeding an intensity $s$ ) is consistent with $S-3 / 2$ which is characteristic of a population that is isotropically distributed in space. So most likely the events are either a local galactic population or extragalactic beyond the local group in origin. If they are local $(100 \mathrm{pc})$ the typical total energy release is $10^{36} \mathrm{erg}$, if they are at a distance of $10 \mathrm{mpc}$ the energy release is $10^{48} \mathrm{erg}$. In any case they are a new phenomenon that was not anticipated. 


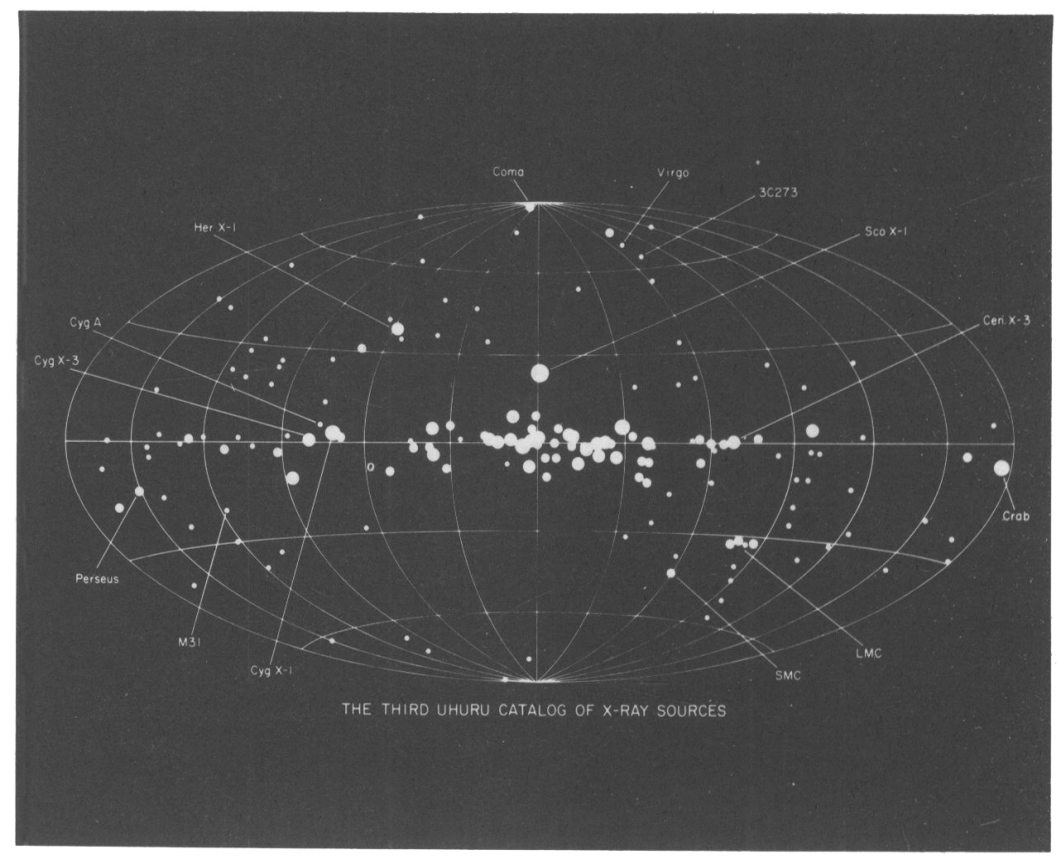

Fig. 1. The third Uhuru catalog of X-ray sources.

\section{INFRARED SPACE ASTRONOMY}

$$
\text { (D. A. Harper) }
$$

The field of infrared astronomy is currently in a state of very rapid development. The opportunities for exploiting the technological advances in infrared instrumentation with groundbased telescopes has commanded the most attention during the last few years. Nevertheless significant progress has been made in the field utilizing high altitude aircraft, balloons, and rockets. Infrared space astronomy is primarily directed towards the studies of sources between $30 \mu$ and $1 \mathrm{~mm}$ where the atmosphere effectively incapacitates large ground-based telescopes. Future infrared space facilities promise higher spatial and spectral resolution. This report briefly outlines those scientific areas of infrared space observations which have been undertaken and describes the techniques employed.

\section{A. Scientific Results}

\section{Broadband Photometry $(30-300 \mu$ region)}

(1) Observations of a large number of luminous sources which emit most of their energy in the far infrared have been investigated. These include:

(a) Compact H II regions and molecular cloud complexes such as W3 and the Orion Nebula, where the $100 \mu$ flux dominates the luminosity.

(b) The Galactic center in which the far infrared flux is shows a different spatial distribution than at $10 \mu$.

(c) Nuclei of active galaxies. The sources observed to date include M82, NGC 253, and NGC 1068.

The luminosities of these sources range from about $5 \times 10^{3}$ to $5 \times 10^{11}$ solar luminosities. The less luminous of these sources are powered by the luminous stars interacting with the 
material in the clouds from which they were formed. The $100 \mu$ flux from the Galactic center can probably be explained by the same process or a similar phenomenon; namely the heating of interstellar dust by the stellar radiation.

Whether or not the sources associated with galactic nuclei can be considered to be a scaled up version of the galactic sources, the observed luminosities imply that the phenomena must have a profound effect on the structure and evolution of the galaxies in which they are located. The phenomena could be similar to those occurring in QSO's which would in turn affect our understanding of the cosmological implication of the QSO observations.

(2) Planetary observations. The most significant results in far infrared photometry in this area is probably the discovery of internal power sources for both Jupiter and Saturn. This data provides essential boundary conditions on theories of the internal structure and evolution of the major planets.

\section{Il. High Resolution Spectra}

These investigations are intrinsically more difficult than wide band observations and developments in this field have proceeded at a somewhat slower pace. The spectral features which have received attention are as follows.

(1) The $28-\mu$ line of $\mathrm{H}_{2}$. Searches for molecular hydrogen at $28 \mu$ have been conducted but to date the line has not been observed.

(2) Features which can be attributed to dust. Such features have been found in the 10 or $20 \mu$ atmospheric windows. There is, however, the possible observation of a water ice feature obtained on a balloon-borne experiment. This result is relatively uncertain.

(3) Atomic cooling lines in $H I I$ regions. There is the possible detection of the $88 \mu$ line of O Il by a group at Cornell under the direction of Martin Harwit. The observations were made with the NASA Lear Jet. High spectral resolution observations, by a group of investigators from France, The Netherlands, and U.K., using the NASA C141 infrared aircraft, have just been completed of the $18-\mu$ Sulfur line and the $88-\mu$ O II line.

(4) Due to instrumental difficulties and residual Telluric absorption the important C II $156-\mu$ cooling line and molecular lines associated with molecular clouds have not been observed as yet. This will be high on the list of priorities for the Shuttle program in the 1980's.

(5) Planetary observations. Work has begun in this area primarily with the $\mathrm{C} 141$ facility by Harold Larson and Uwe Fink. They have observed water vapor and some other molecular species. This work is still in progress.

\section{Background Radiation}

The best work in this area has been carried out by Paul Richards at U C Berkeley and Ray Weiss at MIT. The results indicate a background temperature of $2.7 \mathrm{~K}$, consistent with the microwave results. No spectral features have been observed and to date there is no definitive answer as to the degree of isotropy of this $\mathrm{mm}$ and submillimeter background radiation.

\section{Aircraft}

\section{B. Techniques}

(1) The NASA Lear Jet. This aircraft utilizes a $0.3-\mathrm{m}$ (12 in.) telescope and the observations have been carried out in the $30-300-\mu$ spectral region. Some of the notable things which have been observed with this instrument include the first observations of the internal power of Jupiter and Saturn, early observations of the galactic center, the first observations of galactic $\mathrm{H}$ II regions and the first observations of external galaxies.

(2) The NASA C 141. The modification of this aircraft permit windowless observations with a $.91-\mathrm{m}$ (36 in.) infrared telescope. There is quite a bit of work in progress now. The observations include high spatial resolution observations of the galactic center, galactic H II regions, and nuclei of external galaxies. High resolution in this spectral region corresponds to beam widths down to the order of $20^{\prime \prime}$. Other work includes the observations of the infrared cooling lines in 
H II regions and observations of planetary atmospheres mentioned in the first section.

\section{Balloons}

(1) W. Hoffmann then at the Goddard Space Flight Center flew a .3-m (12 in.) telescope. The notable results were first observations at the galactic center at $100 \mu$ and a partial survey of the galactic plane at $100 \mu$.

(2) A group under the direction of Richard Jennings at the University College of London using a .4-m (16 in.) balloon-borne telescope has made a large number of observations including photometry and mapping of galactic $\mathrm{H}$ II regions and the galactic center.

(3) Harvard-Smithsonian/Arizona Balloon Experiment with a 1-m (40 in.) telescope have been doing about 1 ' resolution photometry and mapping of galactic infrared sources.

(4) Frank Low at the University of Arizona using a $.2-\mathrm{m}$ ( $8 \mathrm{in}$.) telescope has been doing survey work and observations of galactic sources with a $15^{\prime}$ beam (for this aperture of telescope, this is probably the most sensitive experiment so far).

(5) Olthoff and van Duinen of the University of Groningen have been doing survey work with $1 / 2^{\circ}$ beam and a $.3-\mathrm{m}(12 \mathrm{in}$.) telescope.

\section{Rockets}

(1) The best work in this area has been by the AFCRL (Air Force Cambridge Research Laboratories). They have done a 4,11 , and $20-\mu$ sky survey which has covered almost the whole sky. This represents the most complete sky survey to date in the infrared.

(2) The group at Cornell (Jim Houck, Tim Soifer, Judith Pipher) have carried out an IR rocket program. Observations of the galactic center and a number of $\mathrm{H}$ II regions at large beam widths have been obtained (about $1^{\circ}$ ). The wavelengths for this work were 10,20 and $100 \mu$.

\section{Satellites or Spacecraft}

There has been one experiment flown on a classified Air Force spacecraft that carried out a sky survey at 4,10 , and $20 \mu$ which duplicates to some sense the Air Force rocket work, but this has not yet been published. There was an infrared photometer on the Pioneer spacecraft which did 20 and $40-\mu$ photometry work on Jupiter. This has confirmed the internal|power source for Jupiter and they have scanned the spatial structure of the planet at those wavelengths.

\section{Future Experiments}

\section{Large Space Telescope}

As a part of the NASA study on the Large Space Telescope the Infrared Instrument Definition Team has proposed a photometer capable of $10 \%$ wavelength resolution from $1 \mu$ to $2 \mathrm{~mm}$. By virtue of the large aperture and absence of atmospheric seeing this instrument would have superior. spatial resolution and greater sensitivity than other available or proposed instruments for $\lambda<5 \mu$ and for $\lambda \geqslant 100 \mu$.

\section{Space Shuttle Experiments}

There has been a very strong recommendation for a 1 to $11 / 2-\mathrm{m}$ helium cooled telescope. This would represent several orders of magnitude increase in sensitivity over previous experiments and be more sensitive than the LST instrument in the wavelength region from $5 \mu$ to $100 \mu$.

ESA is presently studying a $2.4 \mathrm{~m}$ uncooled infrared telescope for Spacelab. 


\section{Explorer Class Infrared Satellite}

An Explorer class infrared satellite is currently under study. This is a cooperative experiment between NASA and The Netherlands and instrument teams have been selected. This is an experiment which is designed to do a complete full sky survey at wavelengths between 5 and $300 \mu$.

The following 'Observatory Reports' are presented to indicate the activities of satellite space observatories (Copernicus and ANS), an astrophysical space experiment carried out aboard a manned spacecraft (Orion-2), a space research laboratory, and an individual investigator.

\section{APPENDIX I}

\section{REPORT ON COPERNICUS OBSERVATIONS}

$$
\text { (D. G. York) }
$$

The fourth instrument package in the NASA-OAO series, Copernicus, successfully launched on 1972, August 20 is in its fourth year of operation. The Princeton Experiment package consists of a cassegrain telescope with an $80-\mathrm{cm}$ primary mirror, a $7.5-\mathrm{cm}$ secondary, and a Paschen-Rumge spectrometer which utilizes a concave grating to focus the spectrum on a $1-\mathrm{m}$ Rowland circle. Two moveable carriages, each equipped with two photomultipliers scan the spectrum with nominal bandpasses of 0.05 and $0.20 \AA$ shortward of about $1500 \AA$ to the Lyman limit and twice that bandwidth for the longer wavelength pair of tubes. The spacecraft pointing system has proven capable of maintaining the target star within the $0.3^{\prime \prime}$ wide entrance slit.

Overall design and telescope performance are discussed by Rogerson (1963, Space Sci. Rev. 2, 621), Spitzer and Rogerson et al. (1973, A strophys. J. 181, L97), and York (1975, A stronaut. Res, Pergamon Press). The telescope-spectrometer and the telescope have maintained over $98 \%$ availability for scientific observations, while observing constraints have restricted observing efficiency to an average of $33 \%$. After 3.3 years of operation, less than $20 \%$ degradation in spectrometer sensitivity has occurred longward of $1200 \AA$. The high resolution $(0.05 \AA)$ far UV tube, the optical path of which contains three reflective surfaces, has degraded by about one stellar magnitude, at $1000 \AA$. The intermediate resolution tube $(0.2 \AA)$ has degraded by about 1.8 stellar mag. at the same wavelength. For both tubes the degradation decreases smoothly with increasing wavelength to the $20 \%$ previously quoted for $1200 \AA$.

The guidance system initially used was useful down to about $U=5.6 \mathrm{mag}$, whereas the present sensitivity is about $U=5.2 \mathrm{mag}$. On the other hand, two backup guidance modes have been successfully used as faint as $U=6.2 \mathrm{mag}$. The two faintest objects on which guidance has been successful are HD14633 (08 V, $V=7.5 \mathrm{mag}$.) and Nova Cygni (at $V=8.0 \mathrm{mag}$.). By choosing the slit orientation properly, it is possible to observe fainter companions of bright stars (e.g. Sirius B).

The main research programs, for which the telescope-spectrometer was designed are studies of interstellar matter and of $\mathrm{O}$ and $\mathrm{B}$ stellar spectra. A summary of UV interstellar observations has been given by Morton (see the Report of IAU Commission 34). Since 1973, work has been highlighted by further abundance studies; by detailed interpretation of highly excited rotational levels of $\mathrm{H}_{2}$ to yield densities, temperatures, and $\mathrm{UV}$ radiation flux density in interstellar regions with densities of hydrogen nuclei $n_{\mathrm{H}} \geqslant 10 \mathrm{~cm}^{-3}$; and by observations of a hot component in the interstellar gas $\left(3 \times 10^{6} \mathrm{~K}<T<2 \times 10^{5} \mathrm{~K}\right)$. References are given by Morton.

Complete spectroscopic observations of $\mathrm{O}$ and $\mathrm{B}$ stars have been done by the Princeton group for about 65 stars, and specific features have been selected for study in an additional 70 stars. These studies have resulted in detailed information on stellar winds and coronal-type regions. References are given by T. P. Snow in the report prepared by L. Houziaux for Commission 29.

The remainder of the stellar observations, and a few interstellar programs as well, have been carried out by the rather large group of Guest Investigators. The programs are time restricted, so that the observations for any one program are not so extensive, in general, as the programs 
discussed above. However, UV observations for particular stars have complemented our understanding of a number of peculiar systems, and a rather large body of data is accumulating for some specific areas (chromospheres in $G, F, K$, and $M$ stars, where $L y-\alpha \mathrm{Mg}$ II have been studied in about 13 stars; and Be stars, for which about 15 spectra are now available). Detailed references are given by Snow (referenced above), and we restrict ourselves here to noting the scope of the Guest Investigator program.

Between January 1973, and November 1975, we accepted a total of 92 research proposals. The first observations for each program are restricted to twenty four hours of true scanning time, equivalent to use of the instrument for three solar days. Twelve of these programs have been extended and, in these cases, 6-20 days have been devoted to each specific research program. The research involves some 100 astronomers, $65 \%$ of whom are from U.S. institutions, the remaining 35\% include astronomers from 9 countries: Austria, Belgium, Canada, England, Finland, France, Italy, The Netherlands, and the U.S.S.R. NASA provides research funds to staff of American institutions, while programs from non U.S. institutions are carried on on a no-exchange-of-funds basis. Astronomers visit Princeton initially to write detailed spectrometer scanning routines, and at a later date return to perform preliminary analysis and put the data in a form compatible with the data reduction facilities at their home institutions. The initial use of satellite time generally requires that 4 to 6 days of the astronomer's time be spent in Princeton. In most cases, this does not include time required to reduce the data to its final publishable form. Preliminary corrections for particle background and stray light background are provided at Princeton.

A guide for Guest Investigators using the Princeton telescope on the satellite Copernicus was prepared by T.P. Snow of the Princeton University Observatory. This publication is revised periodically and contains appendices giving all the accepted Copernicus Guest Investigator programs, a list of all stars observed as of the date of compilation and references to all papers published or submitted on Copernicus results. The second edition was released in April 1975 .

A recent extension of the Guest Investigator program has involved observations of solar system phenomena, including observations of Mars, Jupiter, Io, comets 1973 VII (Kohoutek), and 1975h, Saturn, Titan, the Ly- $\alpha$ background, and the Earth's atmosphere and geocorona.

Since April 1973, 33\% of the total telescope time has been spent on specific programs for Guest Investigators and time is presently being allocated on the basis of $40 \% /$ year. Thus, Copernicus is an international facility, useful in complementing ground-based research as well as for performing extensive studies of unique physical phenomena.

\section{APPENDIX II}

\section{NETHERLAND ASTRONOMICAL SATELLITE, ANS}

A. UVX Experiment

(Compiled from information provided by

$R$. van Duinen and P. Wesselius)

The ANS-UVX experiment was provided by the Laboratory for Space Research of the University of Groningen. The instrument is essentially a $22-\mathrm{cm}$ telescope with a 5 -channel photometer utilizing grating dispersion and a $2.5^{\prime} \times 2.5^{\prime}$ entrance aperture. The center wavelengths and the bandpass widths are $1550(150), 1800(150), 2200(200), 2500(150)$, and $3295(100)$, all expressed in $\AA$. A preliminary report of the instrument is given by van Duinen et al. (1975, Astron. Astrophys. 39, 159). Up to October 197516000 observations - pointings - were performed by the ultraviolet experiment on a total of 6200 different sky positions. Operations were terminated on 30 November 1975. The number of guest observer programs is approximately 25. Data analysis will continue for some time. Borgman, van Duinen, and Koornneef (1975, Astron. Astrophys. 40,461) presented results on ultraviolet observations of associations in the Large Magellanic Cloud. C. C. Wu has presented a discussion of ultraviolet observations of some close binary systems by ANS at the IAU Symposium No. 
73, 'The Structure and Evolution of Close Binary Systems', 28 July -2 August, Cambridge, England.

The following survey of ANS-UVX observing programs was prepared by P. R. Wesselius.

\section{G: Guest Observer Program}

The number of observations tabulated here may increase because all are not in yet; a large volume of data has not been thoroughly checked yet on its quality.

Program

And romeda Nebula, G

Magnetic Ap and Am Stars, G

Alpha Persei

Be Stars, G

Be Stars, $G$

Subluminous Stars

Beta Canis Majoris Stars, G

Binary System s, g

Cepheus OB3

Calibration Stars

Cepheids, G

Cluster Stars, G

Diffuse Band Stars

Delta S cuti Stars

Extinction Calibration Stars

Elliptical Galaxies

H II Regions in External

Galaxies, $G$

Extinction in Carina Region

Effective Temperature

Extinction

Globular Clusters

Galaxies M81 and M33, G

Galaxies

Galaxies, G

Galaxies, G

Galaxies

Helium Rich Stars, G

Helium Rich Stars

Helium Variable Stars, G

$H$ and Chi Persei

Hyades

Lou Type Stars

Large Magellanic Cloud, G

Stars well observed by OAO-A2

Old Novae

Open Clusters, Integrated Light

Orion Aggregate

O Stars

Stars with known parallax

Photometry in Borgman's system

Pleiades

Planetary Nebulae, G

Praessepe
Person(s) responsible

Borgman

Heuvel

Kester

Savage

Thé and v. Paradijs

Wesselius

Plaut

Kondo, McCloskey, Wu

$v$. Duinen and Blaauw

Aalders

Lub and v. Paradijs

Laval and Laget

Wu, Snow, York

Wesselius

de J onge, UVX group

v. Albada and de Boer

Israel

$v$. Duinen and Vader

Aalders

de Jonge, UVX group

v. Albada and de Boer

Bosma

Wu

de Bruyn

Gallagher, Faber

Wesselius

Klinglesmith

Wu

Molnar

Kester

Kester

UVX group

Borgman, Koornneef

Aalders, Kester

Wesselius

Kester, Borgman, Hartsuiker

v. Duinen and Kester

UVX group

de Boer and Wesselius

UVX group

Kester

Pottasch

Kester
Nr. of

observations

available

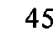

284

114

28

133

481

86

61

139

several

hundred

87

7

73

86

148

45

35

230

5

1511

147

558

26

6

14

213

177

1036

about 2000

46

80

186

396

23

1778

89

114

55 
Program

R-Associations (v.d. Bergh), G

Reflection Nebulae

Reflection Nebulae

Stellar Rotation, G

Run Away Stars

Scorpio-Centaurus Association

Supergiants, $G$

Exciting Stars of H II Regions, G

Sco OB1 association

Stars selected from CSI

T Tauri Stars

Uranus and Neptune, G

Upper Scorpio

Variable Stars

Wolf-Rayet Stars, G

Wolf-R ay Stars

$X-R$ ay Sources

Young Clusters

Zwicky Galaxies
Person(s) responsible

Olth of

And riesse and Witt

de Boer

Peytremann

Wesselius, v. Duinen

Kester, Wesselius

Lub and v. Pradijs

Olth of

Kester, Wesselius

UVX group

de Boer

Savage

Kester, Wu, Wesselius

Wu
Burton

Wu, Hensberge

Wesselius and v. Duinen

Israel
Nr. of

observations

available

10

103

71

228

128

2

57

131

50

42

222

418

137

56

80

\section{B. ANS Soft X-ray Detectors}

(Compiled by C. De Jager)

The Astronomical Netherlands Satellite included among other things two X-ray detectors developed by the Space Research Laboratory of the Astronomical Institute at Utrecht. These instruments are measuring the cosmic X-rays in the range $0.2-0.28 \mathrm{keV}$ and between 1 and $7 \mathrm{keV}$. A description is given by Brinkman et al. (1974a). High-time resolution observations (up to $1 \mathrm{~ms}$ ) and spectral observations are possible.

Heise et al. (1976) were the first to detect X-ray emission from flare stars: an X-ray flare was detected from $\mathrm{YZ} \mathrm{CMi} \mathrm{at} 0.2-0.28 \mathrm{keV}$, but no optical or radio coverage was available. During a very large optical flare from UV Ceti X-ray emission at $0.2-0.28 \mathrm{keV}$ was detected.

Brinkman et al. (1974b) examined the radiation from Cygnus X-1 on the basis of Uhuru data. The analysis shows a strong cor relation between the pulsations in the two energy bins with the low energy pulsation lagging behind the high energy ones.

Heise et al. (1975) showed that from May 1 to May 8, 1975 Cygnus X-1 underwent an upward transition in its intensity, that appears to be the inverse of the downward transition seen by Uhuru in April 1971. The bulk of the increase occurs at low energy, between 1 and $2 \mathrm{keV}$. The intensity increased by a factor of 10 over the November 1974 intensity observed by the Astronomical Netherlands Satellite.

The spectral and intensity variations of Cyg X-3 were observed by ANS and discussed by Brinkman et al. (1976). A number of radio pulsars were examined for pulsed X-ray emission by ANS in the soft X-ray range $(0.2-0.28 \mathrm{keV})$. Except for the Crab no pulsed flux has been found.

Mewe et al. (1976) reported that soft X-rays, presumably originating from stellar coronae were discovered with ANS for Capella ( $\alpha$ Aur) and Sirius B ( $\alpha \mathrm{CMa})$. For 26 other stars upper limits were determined. The X-ray fluxes for $\alpha$ Aur and $\alpha \mathrm{CMa} \mathrm{B}$ are in reasonable agreement with theoretical predictions.

\section{REFERENCES}

Brinkman, A. C. et al.: 1974a, Astrophys. J. 188, 603.

Brinkman, A. C. et al.: 1974b, Philips Technical Rev. 34, 43. 
Brinkman, A. C. et al.: 1976, Space Res. XVI.

Heise, J. et al.: 1975 , submitted to Nature.

Heise, J. et al.: 1976, submitted to Astrophys. J.

Mewe, R. et al.: 1976, Space Res. XVI.

APPENDIX III

REPORT ON ORION-2

(G. A. Gurzadyan)

In December 1973 we conducted an astrophysical experiment by means of the space observatory 'Orion-2' installed aboard the spaceship Soyuz-13. Ultraviolet spectrograms in the region of $2000-5000 \AA$ were obtained for a large number of stars. These spectra were obtained utilizing a wide-angle telescope with a quartz objective prism. We managed to obtain ultraviolet spectrograms, shortward of $3000 \AA$, of rather faint stars - down to $12-13^{m}$ with the exposure time of 18 minutes.

After a year of analysis of the data obtained by means of Orion-2, my colleagues and I are completing the processing and measurements of a few hundred ultraviolet stellar spectra in the wavelength region from $2000-4000 \AA$ and as faint as $13^{\mathrm{m}}$. The following articles published as of January 1975 are:

Gurzadyan, G. A.: Obs. 94. Nature 250, 204, 1974; Monthly Notices Roy. Astron. Soc. (in publ.); Sky Teles. 48, 213, 1974; Astrofizika 10, 379, 1974; Obs. (in publ.); Publ. Astron. Soc. Pacific 87, 289, 1975; Astron. Astrophys. 35, 213. 1974; Space Res. Rev. (in publ.); Astron. Astrophys. 40, 447, 1975; Astron. Astrophys. in publ.); Vestnik Acad. Sci. USSR, No. 1, 1975.

Gurzadyan, G. A. and Ohanesyan, J. B.: Astrofizika. 11, No. 1, 1975.

Gurzadyan, G. A. and Ohanesyan, R. Kh.: Astrofizika 11, No. 2, 1975.

Gurzadyan, G. A. and Rustambekova, S. S.: Nature 254, 311, 1975.

Gurzadyan, G. A. et al.: A strofizika 10, 177, 1974.

The essential part of our results will be presented in the form of four interesting doctoral theses (Ohanesyan, J., Asatryan, R., Ohanesyan, O., Ephremyan, R.); these theses will also be published in the separate volume of Commun. Buyrakan Obs.

As follows even from the subject titles of articles referenced above the results of Orion-2 turned out to be very fruitful. The processings of Orion-2 data are still going on, and we hope to have the new interesting results.

\section{APPENDIX IV}

\section{REPORT OF THE SPACE RESEARCH LABORATORY OF THE ASTRONOMICAL INSTITUTE OF THE STATE UNIVERSITY AT UTRECHT}

\section{(W. de Graaf)}

\section{A. Solar Research}

The hard X-ray solar spectrometer of the Space Research Laboratory of the Utrecht Astronomical Institute, launched on board the ESRO TD-1 A satellite in March, 1972, has continued to function properly till the end of the operational lifetime of the satellite in early May, 1974. Out of several tens of events observed by the instrument, some 10 larger ones were selected for further analysis $(1,2,3)$. This work has resulted in two Doctor's dissertations, of Van Beek (4) and Hoyng (5), respectively.

The Space Research Laboratory of the Utrecht Astronomical Institute is, together with Imperial College, London, and the Space Science Department of ESTEC, Norrdwijk, preparing an instrument for the International Sun-Earth Explorer (ISEE-C), which will be launched in 1978 in a heliocentric orbit near the Earth. The instrument will contain a number of solid state 
detector telescopes, and will observe the spectrum and directional distribution of solar protons with energies between 30-1500 keV.

An instrument to observe the spatial structure, spectrum, and life history of solar flares in the $3.5-20 \mathrm{keV}$ hard X-ray region has been proposed by the Space Research Laboratory of the Utrecht Astronomical Institute for NASA's Solar Maximum Mission. The proposal was selected by NASA for further definition studies.

\section{B. Stellar Ultraviolet A stronomy}

The ultraviolet stellar spectrometer of the Space Research Laboratory of the Utrecht Astronomical Institute, launched on board the ESRO TD-1 A satellite in March, 1972, has continued to function properly till the end of the operational lifetime of the satellite in early May, 1974. Altogether some 3600 spectra of about 200 stars were observed in the wavelength ranges between $2060-2160,2490-2590$, and $2770-2870 \AA$, with a resolving power of $1.8 \AA$. An extensive description of the instrument has been published (6). The results have provided the basis for a number of studies (e.g. 7, 8, 9, 10, 11, 12), as well as for the Doctor's dissertations of Der Boer (13), Lamers (14) and Snijders (15).

A balloon-borne ultraviolet stellar spectrophotometer (BUSS) is being prepared by the Space Research Laboratory of the Utrecht Astronomical Institute and the Astrophysics Section of the L.B. Johnson Space Center, Houston, Texas. The instrument will be capable of recording spectra between $2000-3400 \AA$ with a resolving power of $0.1 \AA$ of stars with magnitude 5 and brighter. The first flights are envisaged to take place in early 1976.

\section{Cosmic X-Ray Astronomy}

The Astronomical Netherlands Satellite (ANS) was successfully launched in August, 1974. It carried one ultraviolet stellar photometry experiment from the Space Research group of the Groningen University, two soft X-ray experiments from the Space Research Laboratory of the Utrecht Astronomical Institute, and one hard X-ray experiment from American Science and Engineering and the Massachusetts Institute of Technology, Cambridge, Massachusetts $(17,18)$. One of the Utrecht experiments consists of a grazing incidence parabolic mirror and a proportional counter system with a polypropylene window, sensitive in the 44-70 $\AA$ wavelength range, and the other Utrecht experiment consists of a collimator and a large area proportional counter system with a titanium window, sensitive in the $1.5-12$ and $27-35 \AA$ wavelength ranges. The short wavelength range is electronically divided in 5 intervals. The position of the sources can be determined to about $0.2 \mathrm{deg}$. Although the orbit of the spacecraft differed considerably from the intended one (altitudes varying between $260-1170 \mathrm{~km}$ instead of between $510-560 \mathrm{~km}$ ), both experiments provided valuable results so far, a number of which were presented at the Eighteenth Meeting of COSPAR, Varna, Bulgaria in 1975 (19). The parabolic detector broke down in June, 1975; the other instruments were still operational (August 1975). See the preceeding report on ANS.

\section{REFERENCES}

1. Hoyng, P. and Stevens, G. A.: 1972, in Xanthakis, J. (ed.), Proc. First European Astron. Meeting, Athens, $1,97$.

2. Brown, J. C. and Hoyng, P.: 1975, Astrophys. J. (in press).

3. Hoyng, P. et al.: 1975, Solar Phys. (in preparation).

4. van Beek, H. F.: 1973, Thesis, Univ. of Utrecht.

5. Hoyng, P.: 1975, Thesis, Univ. of Utrecht.

6. de Jager, C. et al.: 1974, Astrophys. Space Sci. 26, 207.

7. van der Hucht, K. A. and Lamers, H. J.: 1973, Astrophys. J. 181, 537.

8. Lamers, H. J.: 1975, Phil. Trans. Roy. Soc. London A 279, 445.

9. van der Hucht, K. A.: 1975, Phil. Trans. Roy Soc. London A 279, 451.

10. de Jager, C. et al.: 1975, Astrophys. Space Sci. (in press). 
11. Snijders, M. A. J. and Lamers, H. J.: 1975, Astron. Astrophys. (in press).

12. Lamers, J. H. and Snijders, M. A. J.: 1975, Astron. Astrophys. (in press).

13. de Boer, K. S.: 1975, Thesis, Univ. of Groningen.

14. Lamers, H. J.: 1974, Thesis, Univ. of Utrecht.

15. Snijders, M. A. J.: 1975, Thesis, Univ. of Groningen.

16. Bulban, E. J.: 1974, Aviation Week and Space Technology 101, No. 22, 43.

17. van Duinen, R. J. et al.: 1975, Astron. Astrophys. 39, 159.

18. Brinkman, A. C. et al.: 1974, Philips Techn. Rev. 34, 43.

19. various authors 1976, Space Res. 16, (in press).

\section{APPENDIX V}

\section{REPORT FOR COMMISSION 44 FOR THE PERIOD (1973-1975)}

\section{(Y. Kondo)}

During the triennial period 1973-1975, Y. Kondo (Johnson Space Center, Houston) was involved in the following areas of research related to the interests of IAU Commission 44.

(A) Observation of the $\mathrm{Mg}$ II resonance doublet near $2800 \AA$ with a Balloon-borne Ultraviolet Stellar Spectrometer (BUSS) for investigation of stellar atmospheres, particularly chromospheres and other types of extended atmospheres. BUSS was flown very successfully in October 1974 and March 1975. Scientific collaborators included J. Modisette and T. Morgan. High quality results were obtained for 27 stars in the $2275-2825 \AA$ range with a slit width of $0.25 \AA$. Significant results include: (a) strong Mg II doublet emissions in late-type giants and supergiants; (b) study of the cool gaseous shells surrounding late type supergiants $\alpha$ Ori and $\epsilon$ Peg; (c) study of the emission in F-type supergiants $\alpha$ UMi and $\alpha$ Per; (d) mass loss from the A-type supergiants $\alpha$ Cyg and $\eta$ Leo; (e) relationship between the Mg II emission widths and absolute magnitude of the intermediate-to-late-type stars. The BUSS detector system, which had a combination of Ebert-Fastie spectrograph and image dissector tube, is being replaced with a combination of echelle spectrograph and SEC Vidicon system in collaboration with the Space Research Laboratory, Utrecht, the Netherlands. The first flight of the new payload is slated for 1976; the new payload will be capable of observation in the range $2000-3400 \AA$ at a resolution of $0.1 \AA$.

(B) Observation of mass flow in close binary systems and the Mg II doublet features with Copernicus Princeton University telescope spectrometer (as guest investigator): The Copernicus observations of $\beta$ Lyrae obtained in collaboration with M. Hack, J. Hutchings, G. McCluskey and M. Plavec, show that the far ultraviolet spectrum of this binary is completely dominated by emission lines of multiionized elements; the binary is surrounded by high temperature plasma. The observations, with McCluskey, of UW CMa show a large mass loss $\left(3 \times 10^{-6} M_{\odot}\right.$ per year) from this binary; the escaping gaseous cloud surrounds the entire binary system. The Mg II doublet observations, performed with R. Dufour, J. Modisette and G. Wolf, showed evidence of emission in some B-type stars. The equivalent widths of the $\mathrm{Mg}$ II resonance and subordinate lines in some $40 \mathrm{O}$ to $\mathrm{B}$ type stars agree well with the theoretical predictions by Lamers and Snijders except in the earliest spectral types where the rotation effects predicted by Collins appear to become significant.

(C) Collaboration with K. Henize on the SO19 Skylab Ultraviolet Objective Prism Spectrography observations and guest investigator participation in other satellite projects, such as S59 Spectrometer on TD1, ANS Ultraviolet Photometer and OSO-9 French Nighttime Experiment. Some of the results obtained have already been analyzed but most of the data are still in the process of being reduced for interpretation.

(D) A campaign to observe X-ray binaries, supported by IAU Commission 42 and 44; it was initiated in January 1974 with Y. Kondo as its coordinator. Some hundred scientists, including 
$\mathrm{X}$-ray experimenters, ground-observers and theorists, are participating in this campaign. As an outgrowth of this campaign, a 'Symposium on X-Ray Binaries' was organized and held at Goddard Space Flight Center on 20-22 October 1975. Kondo was the chairman of the Scientific Organizing Committee; E. Boldt was co-chairman and also chairman of the Local Organizing Committee. The symposium was attended by some 150 registered participants that are active in this field of research.

A. D. CODE

President of the Commission 